Time was the most critical criterion; only $14 \%$ of the patients were admitted in due time. However, this percentage would probably increase if alteplase were approved, so we also estimated treatment effect assuming the ideal-all patients admitted in due time. In this case 539 patients $(45 \%)$ would have been eligible (figure). Of these patients, 119 died and 150 had full recovery, leaving 270 patients who survived but did not achieve full recovery who could have benefited from treatment. An estimated 48 patients $(150 \times 0.32=48$ (1 to 105$))$, or $4 \%(0.1 \%$ to $8 \%)$ of the stroke population, would have benefited from treatment.

\section{Comment}

Combining data from the US alteplase trial ${ }^{1}$ and the Copenhagen stroke study, we estimated that $0.4 \%$ of unselected stroke patients would benefit from alteplase treatment. With no time constraints for treatment, still only $4 \%$ would benefit. These estimates may be too generous, as we could not exclude patients with rapidly improving symptoms, a criterion excluding $10 \%$ in the US trial. In conclusion, treatment with alteplase may benefit single patients but will have no impact on the general prognosis of stroke. Because time is crucial and because evaluation of patient and paraclinical data requires a specialist setting, treatment with alteplase will need large investments and reorganisation of the care for stroke patients. Before it is decided to offer this expensive, potentially harmful, and possibly only marginally effective treatment we suggest that another, much larger, European trial is needed to test the results of the US trial.

Contributors: HSJ was principal investigator of the Copenhagen stroke study. All the authors participated in designing the study, data collection, and writing this paper. HSJ and TSO analysed and interpreted data for this paper and are guarantors for the paper.

Funding: The study was supported by grants from The Danish Health Foundation and The Danish Heart Foundation.

Competing interests: None declared.

1 National Institute of Neurological Disorders and Stroke rt-PA Stroke Study Group. Tissue plasminogen activator for acute ischemic stroke. $N$ Engl J Med 1995;333:1581-7.

2 The ECASS Study Group. Intravenous thrombolysis with recombinan tissue plasminogen activator for acute hemispheric stroke. JAMA $1995 ; 274 ; 1017-25$

3 The ECASS II Study Group. Randomised double-blind placebocontrolled trial of thrombolytic therapy with intravenous alteplase in acute ischaemic stroke (ECASS II). Lancet 1998;352:1245-51.

4 Jørgensen HS, Nakayama H, Raaschou HO, Olsen TS. Effect of blood pressure and diabetes on stroke in progression. Lancet 1994;344:156-9. (Accepted 18 February 1999)

\title{
Near patient testing for respiratory syncytial virus in paediatric accident and emergency: prospective pilot study
}

\author{
Audrey Mackenzie, Nick Hallam, Elaine Mitchell, Tom Beattie
}

Respiratory syncytial virus is the most important respiratory pathogen in young children, causing bronchiolitis and pneumonia. Infection is especially serious for those who are immunocompromised and those with conditions such as bronchopulmonary dysplasia and congenital heart disease. ${ }^{1}$ The virus is highly infectious, and annual outbreaks cause many hospital admissions, putting great strain on isolation facilities and infection control measures. Nosocomial transmission is well documented. ${ }^{2}$ Rapid testing for the virus is well established $^{3}$ and cost effective. ${ }^{4}$ Near patient testing has the added potential benefits of even faster diagnosis, further cost saving, and improved patient management and infection control. We describe a prospective pilot study of near patient testing for respiratory syncytial virus which was carried out in the accident and emergency department of the Royal Hospital for Sick Children, Edinburgh, between December 1997 and March 1998.

\section{Patients, methods, and results}

One hundred and three pairs of nasopharyngeal secretions were obtained from 98 children under 2 years of age presenting with respiratory symptoms (five children presented twice). The first specimen was sent for direct immunofluorescence testing at the Regional Clinical Virology Laboratory, City Hospital, Edinburgh (accredited by Clinical Pathology Accreditation (UK)) using the
Imagen respiratory syncytial virus reagent (Dako, Ely, United Kingdom). The second specimen (taken immediately afterwards but often smaller in volume) was tested by staff of the accident and emergency department using an enzyme immunoassay (Abbott TestPack RSV, Abbott Laboratories, North Chicago, IL).

This protocol was adopted to avoid compromising the results of direct immunofluorescence testing (our routine method) while the pilot study was in progress. Staff training and near patient testing were carried out in accordance with published guidelines. ${ }^{5}$ Patients with positive results by the near patient test were isolated or put with others with positive results; those with negative results were also isolated if possible while awaiting further results.

The table shows the results for 94 specimen pairs. Results for the other nine specimen pairs are not included (in two cases the results of the near patient test were void, in two cases direct immunofluorescence was unsatisfactory, and in five cases no specimen was

Comparison of results of near patient and direct immunofluorescence testing

\begin{tabular}{lcc} 
& \multicolumn{2}{c}{ Direct immunofluorescence } \\
\cline { 2 - 3 } & Positive & Negative \\
\hline Near patient: & & 1 \\
\hline Positive & 50 & 30 \\
\hline Negative & 13 &
\end{tabular}

Royal Hospital for Sick Children, Edinburgh EH9 1LF

Audrey Mackenzie, clinical nurse specialist

Elaine Mitchell, staff nurse

Tom Beattie, consultant in paediatric accident and emergency medicine

Regional Clinical Virology Laboratory, City Hospital, Edinburgh EH10 5SB

Nick Hallam, consultant virologist

Correspondence to: Dr Hallam

BMJ 1999;319:289-90 
received at the laboratory). Compared with direct immunofluorescence, near patient testing showed a sensitivity of $79 \%$ (95\% confidence interval $67.3 \%$ to $88.5 \%)$, a specificity of $97 \%(82.8 \%$ to $99.9 \%)$, a positive predictive value of $98 \%(89.6 \%$ to $99.9 \%)$, and a negative predictive value of $70 \%$ (53.9\% to $82.8 \%)$.

Near patient testing proved acceptable to the staff performing it, and it was well received in the hospital wards in terms of facilitating patient management and infection control.

\section{Comment}

The high specificity $(97 \%)$ of the enzyme immunoassay in this pilot confirmed results from a laboratory based study. ${ }^{3}$ The comparatively low sensitivity (79\%), although comparable with the sensitivity in another study, ${ }^{3}$ is partially explicable by our using a second, often smaller, specimen for the test. Our results suggest that a positive result of a near patient test is trustworthy and does not require laboratory confirmation (allowing considerable savings in both time and cost) but a negative result needs further investigation. Only one specimen per patient should routinely be required, but this should be treated aseptically to allow for possible laboratory referral.

Near patient testing for respiratory syncytial virus is a viable option for paediatric accident and emergency with important potential benefits.
Contributors: AM designed the study and oversaw the near patient testing. $\mathrm{NH}$ assisted in the study design, oversaw near patient and laboratory testing, and wrote the manuscript. EM oversaw and performed near patient testing. TB assisted in the study design and in writing the manuscript. Staff of the accident and emergency department, Royal Hospital for Sick Children, Edinburgh, and of the Regional Clinical Virology Laboratory, City Hospital, Edinburgh, provided technical help. Dr Peter Mackie (who has pioneered the concept of near patient testing for respiratory syncytial virus) and Pamela Joannidis (infection control nurse) at Yorkhill Children's Hospital, Glasgow, provided helpful advice. NH is guarantor for the paper.

Funding: Pump priming, Royal Hospital for Sick Children, Edinburgh.

Conflict of interest: None declared.

1 Wendt $\mathrm{CH}$, Hertz MI. Respiratory syncytial virus and parainfluenza virus infections in the immunocompromised host. Semin Respir Infect $1995 ; 10: 224-31$.

2 Langley JM, LeBlanc JC, Wang EEL, Law BJ, MacDonald NE, Mitchell I, et al. Nosocomial respiratory syncytial virus infection in Canadian pediatric hospitals: a Pediatric Investigators Collaborative Network on Infections in Canada study. Pediatncs 1997;100:943-6.

3 Todd SJ, Minnich L, Waner JL. Comparison of rapid immunofluorescence procedure with TestPack RSV and Directigen FLU-A for diagnosis of respiratory syncytial virus and influenza virus. J Clin Microbiol 1995;33:1650-1.

4 Woo PCY, Chiu SS, Seto W-H, Peiris M. Cost-effectiveness of rapid diagnosis of viral respiratory tract infections in paediatric patients. J Clin Microbiol 1997;35:1579-81.

5 Joint Working Group on Quality Assurance. Guidelines on near to patient or point of care testing. Liverpool: Joint Working Group on Quality Assurance, 1999. (Available from Diagnostics Services, Mast House, Liverpool L20 1EA.)

(Accepted 5 March 1999)
National Addiction Centre, The

Maudsley Hospital and Institute of Psychiatry, London SE5 8AF

David Best, research coordinator

Alison Noble,

researcher

Emily Finch, consultant psychiatrist Michael Gossop head of research (addiction directorate

Clare Sidwell, researcher

John Strang

director

Correspondence to: D Best

d.best@iop.kcl.ac.uk

BMJ 1999;319:290-1
Infection with hepatitis $\mathrm{C}$ and hepatitis B viruses is common among injecting drug users. ${ }^{1-4}$ In a sample of blood donors only $0.01 \%$ were positive for antibodies to hepatitis $\mathrm{C}$ virus, ${ }^{5}$ whereas $59 \%$ of injecting drug users in East Anglia were positive, with $22 \%$ also positive for hepatitis B virus. ${ }^{2}$ Rhodes et al reported that about half (58\% in 1992 and 50\% in 1993) of those whose salivary specimens contained antibodies to hepatitis B virus were unaware of their infection. ${ }^{4}$ Although drug users are generally aware of the risks of infection, their awareness of their own status is uncertain. We tested opiate addicts receiving methadone maintenance treatment for markers of hepatitis B and hepatitis $\mathrm{C}$ infection and compared the results with their beliefs about their viral status.

\section{Subjects, methods, and results}

We collected data on 106 injecting opiate addicts attending a methadone maintenance clinic in London. Sufficient blood was obtained for 90 hepatitis C virus tests and for 84 hepatitis $\mathrm{B}$ virus tests (when blood was insufficient, priority was given to hepatitis $\mathrm{C}$ ). We present data for the 90 addicts from whom blood was obtained.

The participants' mean age was 36 years (range 21-54), and 69 were men. They had attended the clinic for an average of 2.4 years and received a mean methadone dose of $72 \mathrm{mg}$ (range 15-150 mg). Their mean age at first opiate injection was 21 , and the mean duration of injecting was 15 years. Fifty nine had used a syringe after someone else, and 68 had shared injecting paraphernalia.

Seventy seven were positive for hepatitis $\mathrm{C}$ virus, and 46 were positive for hepatitis $\mathrm{B}$ virus. Of the 79 who reported having previously been tested for hepatitis $\mathrm{C}$ virus, 58 thought they were positive, 16 thought they were negative (of whom half were wrong), and five were uncertain. Of the 70 addicts previously tested for hepatitis $\mathrm{C}$ virus whom we found to be positive, $12 \mathrm{did}$ not know or were wrong about their status (see table). Of the 41 previously tested for hepatitis B virus whom we found to be positive, 16 thought themselves to be negative.

Of the 11 addicts never previously tested for hepatitis $\mathrm{C}$ virus, four were correct in their self assessment (one positive, three negative), four incorrectly thought they were negative, and three did not know. Of the 22 addicts not previously tested for hepatitis B virus, six were correct in their self assessment (all negative), while three mistakenly believed they were positive and one negative, and 12 could not report their viral status (five positive, seven negative). 